\title{
Evidence for anti-angiogenic and pro-survival functions of the cerebral cavernous malformation protein 3
}

\author{
Elisa Schleider • Sonja Stahl • Joycelyn Wüstehube • \\ Ulrich Walter • Andreas Fischer • Ute Felbor
}

Received: 6 May 2010 /Accepted: 3 September 2010 /Published online: 23 September 2010

(C) The Author(s) 2010. This article is published with open access at Springerlink.com

\begin{abstract}
Mutations in $C C M 1, C C M 2$, or $C C M 3$ lead to cerebral cavernous malformations, one of the most common hereditary vascular diseases of the brain. Endothelial cells within these lesions are the main disease compartments. Here, we show that adenoviral CCM3 expression inhibits endothelial cell migration, proliferation, and tube formation while downregulation of endogenous CCM3 results in increased formation of tube-like structures. Adenoviral
\end{abstract}

Electronic supplementary material The online version of this article (doi:10.1007/s10048-010-0261-6) contains supplementary material, which is available to authorized users.

E. Schleider $\cdot$ S. Stahl

Department of Human Genetics, University of Würzburg,

Würzburg, Germany

E. Schleider $\cdot$ S. Stahl $\cdot$ U. Walter

Institute of Clinical Biochemistry and Pathobiochemistry,

University of Würzburg,

Würzburg, Germany

J. Wüstehube $\cdot$ A. Fischer

Joint Research Division Vascular Biology of the Medical Faculty

Mannheim (CBTM), Heidelberg University,

Mannheim, Germany

J. Wüstehube $\cdot$ A. Fischer

German Cancer Research Center (DKFZ-ZMBH Alliance),

Heidelberg, Germany

U. Felbor $(\bowtie)$

Institute of Human Genetics, University Hospital Greifswald,

Fleischmannstr. 42/44,

17475 Greifswald, Germany

e-mail: felbor@uni-greifswald.de

U. Felbor

Interfaculty Institute for Genetics and Functional Genomics,

Division of Human Genetics,

Ernst-Moritz-Arndt University Greifswald,

Greifswald, Germany
CCM3 expression does not induce apoptosis under normal endothelial cell culture conditions but protects endothelial cells from staurosporine-induced cell death. Tyrosine kinase activity profiling suggests that CCM3 supports PDPK-1/ Akt-mediated endothelial cell quiescence and survival.

Keywords Cerebral cavernous malformation · CCM3 · PDCD10

Cerebral cavernous malformations (CCM) have a prevalence of about one in 200 individuals and can occur sporadically or in an autosomal dominantly inherited manner with $60 \%$ disease penetrance. Affected patients present with chronic headaches, epilepsy, or hemorrhagic stroke. Second hit somatic loss-of-function mutations in CCM1 (KRIT1), CCM2 (OSM), or CCM3 (PDCD10) have been identified within cavernous lesions [1].

The gene products of CCM1 and CCM2 were shown to be important for endothelial cell-cell junction formation and maintenance of endothelial barrier function $[2,3]$. Most recently, it could be demonstrated that CCM1 also inhibits endothelial proliferation, apoptosis, migration, lumen formation, and sprouting angiogenesis in primary human endothelial cells [4]. In contrast, CCM3 remains without precise endothelial cell function description. Originally, CCM3 mRNA was found to be upregulated in an apoptotic human myeloid cell line [5]. An apoptosis-inducing role was suggested based on CCM3 overexpression experiments in HeLa cells and siRNA-mediated inhibition of endogenously increased CCM3 in serum-deprived human umbilical vein endothelial cells (HUVECs) [6]. In contrast, CCM3 was reported to promote cell proliferation in a human prostate cancer cell line [7]. Endothelial cell-specific 
disruption of $c \mathrm{~cm} 3$ in mice demonstrated that CCM3 is essential for early embryonic vascular development and acts through stabilization of VEGFR2 signaling [8].

The aim of this study was to characterize CCM3 function in primary human endothelial cells. The isolation of highly pure endothelial cells from CCM lesions has been hampered by calcification of the resected lesions and the presence of diverse cell types within CCM specimen including not only cavernous but also normal neoangiogenic endothelial cells [1]. Therefore, an adenoviral approach with almost 100\% transduction efficiency was used for overexpression of human CCM3 in HUVECs (details are provided in Supplementary Information (ESM 1)).

Adenoviral CCM3 overexpression did not result in a significant proportion of cells with fragmented nuclear morphology indicative of apoptosis as has been reported by Chen et al. for CCM3-transfected HeLa cells [6] (Fig. 1a). However, overexpression of CCM3 led to twofold reduction of cell proliferation, at least twofold reduced motility, and a decreased ability to form complete capillary-like structures on Matrigel. CCM3 depletion resulted in increased tube formation when compared to siRNA control and GFP-overexpressing cells (Fig. 1a-e, for details see Supplementary Information (ESM 1)). This observation that downregulation of endogenous CCM3 results in increased formation of tube-like structures is in agreement with previous ccm 3 knockdown studies in zebrafish that revealed excessive disorganized sprouting of subintestinal vessels [9] identical to the vascular phenotype seen in $\mathrm{ccm} 1$ and $c c m 2$ mutant zebrafish [10]. Given that CCM1, CCM2, and CCM3 form a protein complex in vitro [11], our data are also in line with CCM1 being a negative regulator of sprouting angiogenesis [4].

Similarly, it was recently shown that CCM1 shifts the balance from ERK-mediated proliferation to Akt-mediated cell survival and endothelial quiescence [4]. Accordingly, CCM3-transduced endothelial cells showed decreased metabolic activity which we interpret to be a sign of endothelial quiescence rather than cell death (Fig. 1f).

As observed for CCM1 [4], adenoviral CCM3 expression per se did not induce apoptosis under normal endothelial cell culture conditions (Fig. 1g). However, similar to CCM1, CCM3 reduced the rate of staurosporine-induced cell death: induction of apoptosis with $250 \mathrm{nmol} / \mathrm{l}$ staurosporine for $2 \mathrm{~h}$ resulted in more than fourfold increased caspase 3/7 activities in GFP-overexpressing HUVECs. In contrast, CCM3-overexpressing HUVECs showed only a less than twofold increase in caspase 3/7 activities (Fig. 1g). These data are in line with the observation by Chen and coworkers that endogenous CCM3 expression is increased in HUVECs $30 \mathrm{~min}$ after induction of apoptosis by serum deprivation prior to the increase of cleaved caspase three levels seen after $3 \mathrm{~h}$ [6]. However, our data demonstrate that upregulation of
Fig. 1 Overexpression of CCM3 in HUVECs strongly impaired endothelial cell proliferation, migration, tube formation, and apoptosis and activated the PDPK-1/Akt survival pathway. a HUVECs overexpressing GFP or CCM3 $48 \mathrm{~h}$ after transduction and western blot analysis with anti-CCM3 antibody revealing prominent overexpression of CCM3 (lane 3) and successful downregulation of endogenous CCM3 (lane 4). b Cells overexpressing CCM3 (upper micrograph, upper line, right wells) proliferated half as much as control cells (upper micrograph, upper line, left and middle wells; lower line, middle wells) and siCCM3-transfected cells (upper micrograph, lower line, left wells). c The ability of endothelial cells to migrate was strikingly reduced in cells overexpressing CCM3 (lane 2) when compared to untreated (lane 1) and GFP-transduced cells (lane 3). d, e CCM3-transduced cells showed decreased ability to form complete capillary-like structures (d, lane 3; e, left panel). CCM3-depleted cells showed a slight but significant increase in tube formation (d, lane 4; $\mathbf{e}$, right panel). f CCM3-transduced cells showed decreased metabolic activity using WST-1 reagent assay compared to GFP-transduced cells starting to get obvious $2 \mathrm{~h}$ after incubation. g Overexpression of CCM3 had no effect on apoptosis under normal growth factor conditions, but staurosporine-induced cell death was more than twofold less in CCM3-overexpressing cells when compared to GFPoverexpressing cells. h Western blot analyses of HUVEC lysates overexpressing GFP (lane 1) or CCM3 (lane 2), respectively. Protein expression was detected using anti-phospho-PDPK-1 (Tyr 9), antiphospho-Akt (Thr 308), and anti-phospho-Akt (Ser 473). Anti-Akt staining served as loading control. Phosphorylation of PDPK-1 and Akt was significantly elevated in CCM3-overexpressing cell lysates (lane 2). $* p<0.01$

CCM3 protects endothelial cells from staurosporine-induced cell death rather than inducing it.

Tyrosine kinase activity profiling represents an unbiased novel approach to dissect the mechanisms behind the observed negative effects of CCM3 overexpression on endothelial cell proliferation, migration, tube formation, and apoptosis. Details on tyrosine kinase activity profiling are provided in Supplementary Information (ESM 1). HUVECs transduced with CCM3 demonstrated significantly increased tyrosine 9-specific phosphorylation for 3phosphoinositide-dependent protein kinase 1 (PDPK-1, data not shown). Elevated levels of PDPK-1 phosphorylated at its tyrosine- 9 residue could be confirmed with phosphospecific antibodies against PDPK-1 in HUVECs overexpressing CCM3 (Fig. 1h). PDPK-1 had been shown to be important for endothelial cell migration [12] and formation of a circulatory system in embryonic mice [13] and plays a central role in Akt activation upon growth factor stimulation [14]. In accordance with the observation that embryonic CCM3 knockout tissues showed decreased Akt phosphorylation [8], CCM3 overexpression led to increased phosphorylation of the potent inhibitor of apoptosis Akt at threonine 308 and serine 473 (Fig. 1h) [15]. Again, our data are in line with the fact that CCM1 expression increased the amount of phosphorylated Akt at serine 473 [4]. Thus, CCM3 expression resulted in altered phosphorylation patterns in endothelial cells including activation of Akt and its upstream activator PDPK-1. 


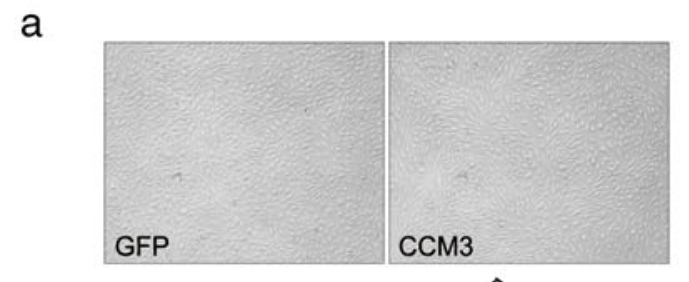

b

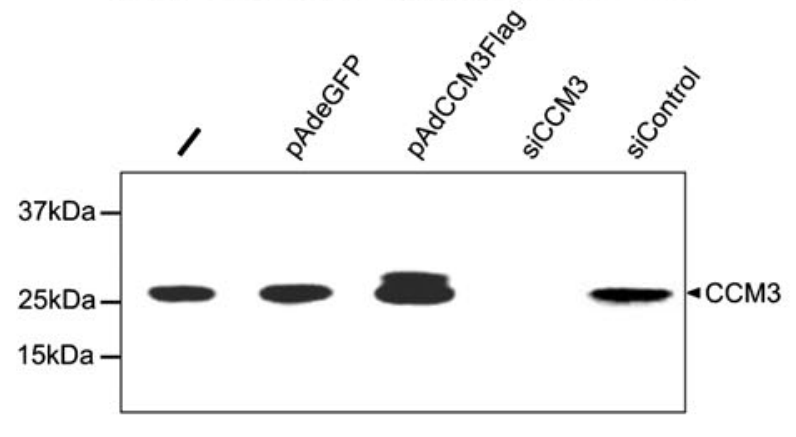

C

d
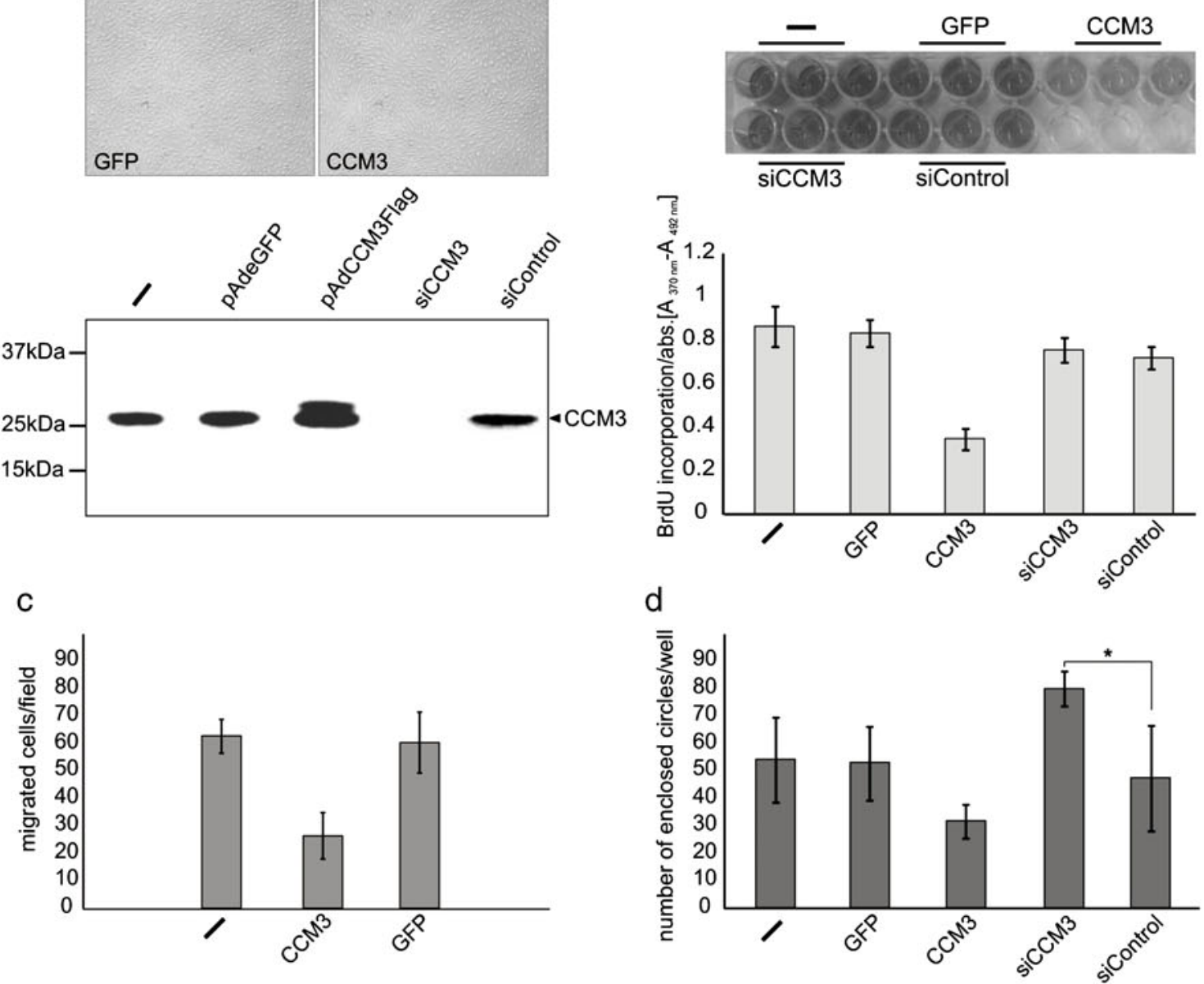

e

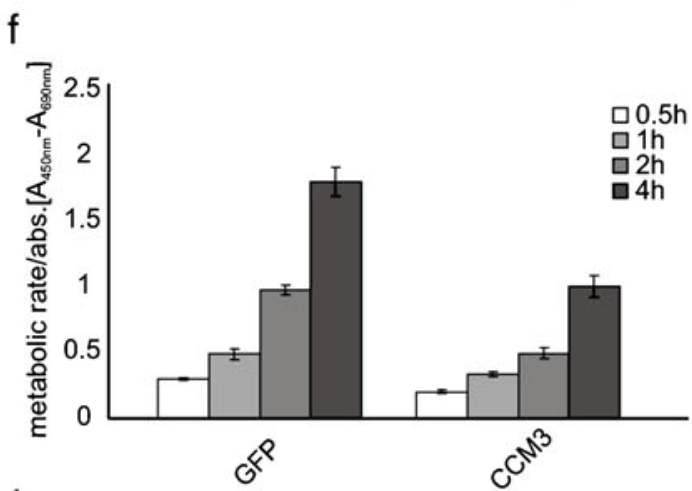

g

h
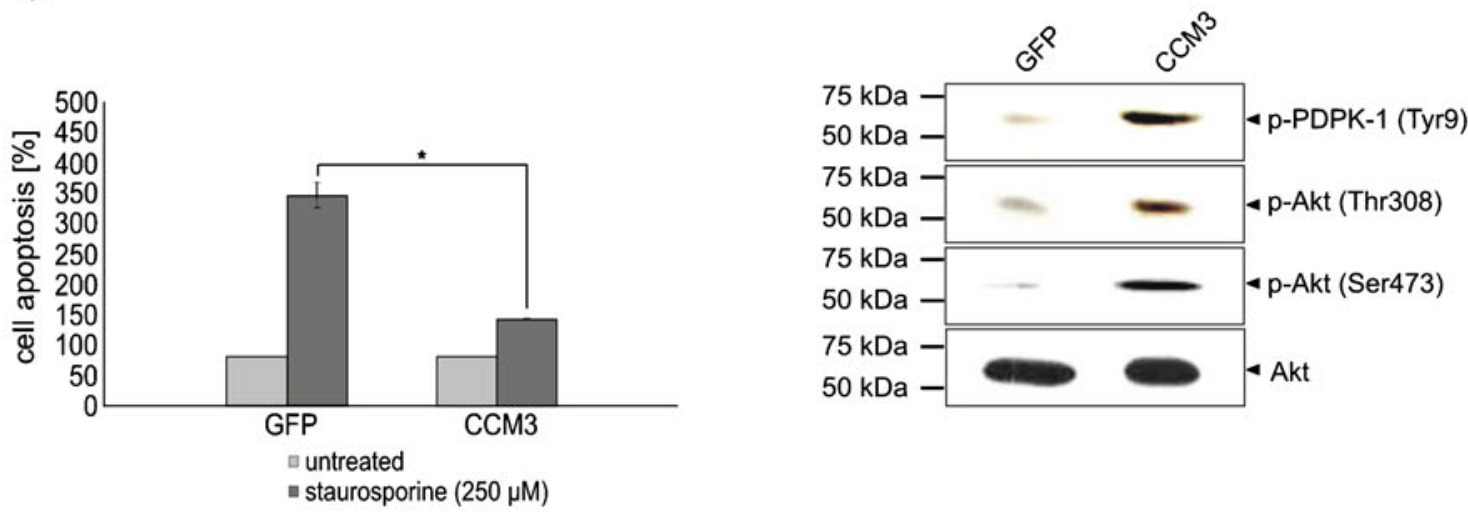
Taken together, we show that CCM3 inhibits endothelial cell migration, proliferation, and tube formation and supports PDPK-1/Akt-mediated endothelial cell survival. Our data suggest that CCM3 plays a crucial role in maintaining endothelial integrity and protection from cell death.

Acknowledgements The authors thank D. Pijnenburg and A. Brilleman from PamGene International B.V. for performing PamChip kinase arrays and bioinformatics. This work was supported by the Bavarian Genome Network. E.S. received a stipend from the Graduiertenkolleg 1048. A.F. receives funding from the Deutsche Forschungsgemeinschaft DFG (SFB-Transregio 23 and Graduiertenkolleg 880).

Open Access This article is distributed under the terms of the Creative Commons Attribution Noncommercial License which permits any noncommercial use, distribution, and reproduction in any medium, provided the original author(s) and source are credited.

\section{References}

1. Limaye N, Boon LM, Vikkula M (2009) From germline towards somatic mutations in the pathophysiology of vascular anomalies. Hum Mol Genet 18:R65-R74

2. Glading A, Han J, Stockton RA, Ginsberg MH (2007) KRIT-1/ CCM1 is a Rap1 effector that regulates endothelial cell cell junctions. J Cell Biol 179:247-254

3. Whitehead KJ, Chan AC, Navankasattusas S, Koh W, London NR, Ling J, Mayo AH, Drakos SG, Jones CA, Zhu W, Marchuk DA, Davis GE, Li DY (2009) The cerebral cavernous malformation signaling pathway promotes vascular integrity via Rho GTPases. Nat Med 15:177-184

4. Wüstehube J, Bartol A, Liebler SS, Brütsch R, Zhu Y, Felbor U, Sure U, Augustin HG, Fischer A (2010) Cerebral cavernous malformation protein CCM1 inhibits sprouting angiogenesis by activating DELTA-NOTCH signaling. Proc Natl Acad Sci USA 107:12640-12645
5. Wang Y, Liu H, Zhang Y, Ma D (1999) cDNA cloning and expression of an apoptosis-related gene, human TFAR15 gene. Sci China (Ser C) 42:323-329

6. Chen L, Tanriover G, Yano H, Friedlander R, Louvi A, Gunel M (2009) Apoptotic functions of PDCD10/CCM3, the gene mutated in cerebral cavernous malformation 3. Stroke 40:1474-1481

7. Ma X, Zhao H, Shan J, Long F, Chen Y, Chen Y, Zhang Y, Han X, Ma D (2007) PDCD10 interacts with Ste20-related kinase MST4 to promote cell growth and transformation via modulation of the ERK pathway. Mol Biol Cell 18:1965-1978

8. He Y, Zhang H, Yu L, Gunel M, Boggon TJ, Chen H, Min W (2010) Stabilization of VEGFR2 signaling by cerebral cavernous malformation 3 is critical for vascular development. Sci Signal. doi:10.1126/scisignal.2000722

9. Voss K, Stahl S, Hogan BM, Reinders J, Schleider E, SchulteMerker S, Felbor U (2009) Functional analyses of human and zebrafish 18-amino acid in-frame deletion pave the way for domain mapping of the cerebral cavernous malformation 3 protein. Hum Mutat 30:1003-1011

10. Hogan BM, Bussmann J, Wolburg H, Schulte-Merker S (2008) ccm 1 cell autonomously regulates endothelial cellular morphogenesis and vascular tubulogenesis in zebrafish. Hum Mol Genet 17:2424-2432

11. Stahl S, Gaetzner S, Voss K, Brackertz B, Schleider E, Sürücü O, Kunze E, Netzer C, Korenke C, Finckh U, Habek M, Poljakovic Z, Elbracht M, Rudnik-Schöneborn S, Bertalanffy H, Sure U, Felbor U (2008) Novel CCM1, CCM2, and CCM3 mutations in patients with cerebral cavernous malformations: in-frame deletion in $\mathrm{CCM} 2$ prevents formation of a CCM1/CCM2/CCM3 protein complex. Hum Mutat 29:709-717

12. Primo L, di Blasio L, Roca C, Droetto S, Piva R, Schaffhausen B, Bussolino F (2007) Essential role of PDK1 in regulating endothelial cell migration. J Cell Biol 176:1035-1047

13. Lawlor MA, Mora A, Ashby PR, Williams MR, Murray-Tait V, Malone L, Prescott AR, Lucocq JM, Alessi DR (2002) Essential role of PDK1 in regulating cell size and development in mice. EMBO J 21:3728-3738

14. Bayascas JR (2008) Dissecting the role of the 3-phosphoinositidedependent protein kinase-1 (PDK1) signalling pathways. Cell Cycle 7:2978-2982

15. Hemmings BA (1997) Akt signaling: linking membrane events to life and death decisions. Science 275:628-630 